\title{
An Asymptotic Optimality of the Transposition Rule for Linear Lists
}

\author{
David Gamarnik and Petar Momčilović \\ IBM T.J. Watson Research Center \\ Yorktown Heights, NY 10598 \\ \{gamarnik, petar\}@us.ibm.com
}

December 21, 2018

\begin{abstract}
The transposition rule is an algorithm for self-organizing linear lists. Upon a request for a given item, the item is transposed with the preceding one. The cost of a request is the distance of the requested item from the beginning of the list. An asymptotic optimality of the rule with the respect to the optimal static arrangement is demonstrated for two families of request distributions. The result is established by considering an associated constrained asymmetric exclusion process.
\end{abstract}

Keywords: self-organizing list, average-case analysis, exclusion process

\section{Introduction}

The linear list is one of basic data structures in computer science with search being a primary operation defined on it. Items are located in the list by sequentially examining them from the beginning of the list. Intuitively one would like to place items that are frequently requested at the front of the list in order to minimize the number of items being examined. Given the properties of the request sequence one could place items in an order that minimizes the search cost. Yet often properties of the request sequence are either not known in advance or time dependent. Hence, it is desirable to employ self-organizing algorithms. The two best known such rules are the move-to-front and transposition rule [9, Section 6]. In addition to being simple these rules are memory-free, i.e., require no memory for their operation.

List organizing algorithms have been analyzed over the past fifty years, e.g., see review on self-organizing linear search in [6]. While the literature on the move-to-front rule (and the corresponding least-recently-used algorithm) is extensive (see, e.g., 3, 4, 5, 7, 12] and references therein), the results on the transposition rule are scarce. Early analysis of the transposition rule can be found in [11. In the same paper it was conjectured that the transposition rule is optimal with respect to the expected value of the search cost. However, in [1] it was shown this conjecture is not true in general. Besides the mentioned papers, probabilistic analysis of the transposition rule is either limited to simplistic distributions [8, 13] or numerical studies [2, 10]. For the combinatorial (amortized) analysis of the transposition rule the reader is referred to [12].

In the present paper we establish an asymptotic optimality of the transposition rule with respect to two families of request distributions. Specifically, we prove that the logarithm of 
the tail probability of the search cost is asymptotically optimal when the transposition rule is used. Our result is obtained by bounding the performance of the transposition rule by a certain constrained asymmetric exclusion process.

The rest of the paper is organized as follows. Model description and main results is stated in the next section. Section 3 describes the asymmetric exclusion process, where a closed form solution is obtained for the limiting behavior. Section 4 relates the particle system to the transposition rule for self-organizing lists. Section [5 contains the proof of the main results. Conclusions and some open questions are in Section 6.

\section{Model and main results}

We consider an infinite list of items $L=\{1,2, \ldots, N, \ldots\}=\mathbb{N}$. At integer times $t=$ $0,1,2, \ldots$ a request arrives for an item from $L$. The item requested at time $t$ is denoted by $R(t)$. Requests are independent and identically distributed with $\pi_{i}$ being the probability of requesting item $i, \sum_{i>1} \pi_{i}=1$. Moreover, without loss of generality we assume that $\pi_{i}>\pi_{i+1}$ for all $i$. Let $R$ be equal in distribution to $R(t)$, i.e., $\mathbb{P}[R=i]=\pi_{i}$.

The evolution of list $L$ is governed by the transposition rule. At time $t=0$ the list is assumed to be ordered as $\{1,2, \ldots, N, \ldots\}$. Upon a request, the requested object is moved forward by one position in the list while the one in front of it is moved one position back. If the first item in $L$ is requested, the list does not change.

Let $X_{i}(t)$ be the position of the item $i$ in the list at time $t$. Our focus is on the behavior of the position $C(t):=X_{R(t)}(t)$ of the requested item, i.e., the search cost, as $t \rightarrow \infty$. We note that if a permutation $\sigma: \mathbb{N} \rightarrow \mathbb{N}$ (one-to-one mapping) is fixed, then the distribution of $C(t)$ is determined completely by $\pi:=\left\{\pi_{i}\right\}_{i=1}^{\infty}$. In that case we use $C^{\sigma}$ to denote the random position of the selected element $R$. Our first result is a simple observation that for every permutation the tail asymptotics of $C^{\sigma}$ dominates the tail asymptotics of $R$.

Lemma 1. For any distribution $\pi$, permutation $\sigma$, and for every $x \in \mathbb{N}$

$$
\mathbb{P}\left[C^{\sigma}>x\right] \geq \mathbb{P}[R>x] .
$$

Proof. One has

$$
\begin{aligned}
\mathbb{P}\left[C^{\sigma}>x\right] & =\sum_{j: \sigma(j)>x} \pi_{j} \\
& \geq \sum_{j>x} \pi_{j}=\mathbb{P}[R>x],
\end{aligned}
$$

where the inequality is due to the monotonicity of $\pi$.

Thus, as far as the tail probability asymptotics is concerned no list ordering algorithm can achieve a better tail asymptotics than $\mathbb{P}[R>x]$.

We say that $R$ is distributed as a power law with parameter $\alpha>1$ if $\pi_{i}=c i^{-\alpha}$ for all $i$, where $c=\sum_{i>1} i^{-\alpha}$ is the normalization constant. Also, $R$ is defined to be asymptotically geometric with parameter $0<\nu<1$ if $i^{-1} \log \pi_{i} \rightarrow \log \nu$ as $i \rightarrow \infty$. Our main result states that the transposition rule is asymptotically optimal with respect to the logarithm of the tail asymptotics for these two distribution families. 
Theorem 1. Let $\pi$ be either power law with parameter $\alpha>1$ or asymptotically geometric with parameter $0<\nu<1$. Then

$$
\varlimsup_{x \rightarrow \infty} \varlimsup_{t \rightarrow \infty} \frac{\log \mathbb{P}[C(t)>x]}{\log \mathbb{P}[R>x]}=1 .
$$

Proof. See Section 5 .

An important special case is when $\pi$ has a finite support. Although we will not make use of the following fact, we note that for every distribution $\pi$ with finite support on $\{1,2, \ldots, N\}\left(\pi_{i}>0\right.$ for $\left.i=1, \ldots, N\right)$ the described system is reversible irreducible aperiodic Markov chain, and the unique stationary solution is of the following product form

$$
\mathbb{P}\left[X_{1}=i_{1}, X_{2}=i_{2}, \ldots, X_{N}=i_{N}\right]=\frac{\prod_{j=1}^{N} \pi_{j}^{-i_{j}}}{\sum_{\left(k_{1}, \ldots, k_{N}\right) \in \Pi_{N}} \prod_{j=1}^{N} \pi_{j}^{-k_{j}}},
$$

where $\Pi_{N}$ denotes the set of all permutations of list $L_{N}=\{1,2, \ldots, N\}$. A natural way to introduce a power law or geometric distribution for the case of finite support is to take the distribution $\pi$ conditioned on event $\{i \leq N\}$. Denote by $\pi_{N}$ the truncated distribution and let random variable $R_{N}$ be defined by $\mathbb{P}\left[R_{N}>x\right]:=\mathbb{P}[R>x \mid R \leq N]$. Our main result carries on to the case of these truncated distributions as well. Note that the existence of a unique stationary distribution allows us to consider the stationary cost denoted by $C_{N}$.

Theorem 2. Let either (i) $\pi_{N}$ be truncated power law with parameter $\alpha>1$ and $x / N<\gamma$ for some $\gamma<1$ or (ii) $\pi_{N}$ be truncated asymptotically geometric with parameter $0<\nu<1$ and $x<N$. Then

$$
\lim _{\{x, N\} \rightarrow \infty} \frac{\log \mathbb{P}\left[C_{N}>x\right]}{\log \mathbb{P}\left[R_{N}>x\right]}=1
$$

Proof. See Section 5 ,

\section{Constrained asymmetric exclusion process}

In the present section we consider a constrained asymmetric exclusion process in which we examine the deviation of the boundary particle from its minimal position. In particular, we consider an $n$ particle system on countably many slots on a half-line enumerated from left to right as $1,2, \ldots$ Each particle is associated with an independent Poisson process of unit intensity (the actual rate is not important). At the instances of their corresponding Poisson processes particles move left or right with probabilities $p$ and $q$, respectively. Multiple occupancies are not allowed and a move actually occurs only if the target slot is empty (see Fig. 1 for an example). Also a particle can not move left if it is located in the first slot.

Assume that $p>q$ and define $\beta:=q / p<1$. Let $Z_{i}$ be the position (slot number) of the $i$-th particle, where particles are enumerated from left to right. We first verify in a very straightforward way that the stationarity solution is of the following form

$$
\mathbb{P}\left[Z_{1}=i_{1}, Z_{2}=i_{2}, \ldots, Z_{n}=i_{n}\right]=\eta_{n}^{-1} \beta^{\sum_{j=1}^{n} i_{j}},
$$

for all $1 \leq i_{1}<i_{2}<\cdots<i_{n}$, where $\eta_{n}$ is the normalizing constant. To this end

$$
\begin{aligned}
\sum_{1 \leq i_{1}<i_{2}<\cdots<i_{n}} \beta^{\sum_{j=1}^{n} i_{j}} & <\sum_{1 \leq j \leq n} \sum_{1 \leq i_{j}<\infty} \beta^{\sum_{j=1}^{n} i_{j}} \\
& =\left(\frac{\beta}{1-\beta}\right)^{n}<\infty
\end{aligned}
$$




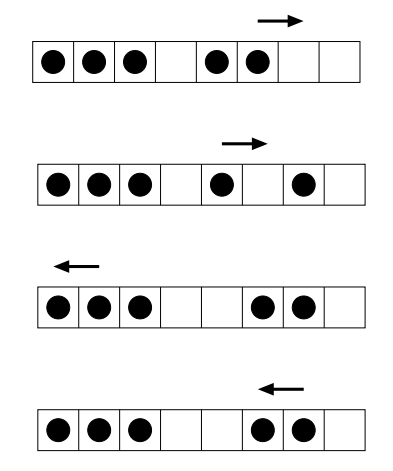

Figure 1: An example of evolution of the system with 4 particles. Arrows indicate intended movements of particles. Movements actually occur only in the first two instances.

and therefore the normalizing constant $\eta_{n}$ is finite. It is straightforward to check that the underlying Markov chain is irreducible, reversible, aperiodic and that (4) satisfies the stationarity equation. Therefore (4) indeed describes the stationary distribution.

We point out that the minimal possible value of $\sum_{i=1}^{n} Z_{i}$ is $n(n+1) / 2$. Throughout the paper we interpret $\prod_{i=j}^{k}(\cdot) \equiv 1$ for $k<j$.

Lemma 2. The normalizing constant satisfies

$$
\eta_{n}=\beta^{\frac{n(n+1)}{2}} \prod_{i=1}^{n} \frac{1}{1-\beta^{i}} .
$$

Proof. For each integer $k \geq 0$ let $\eta_{n, k}$ denote the sum of $\beta^{\sum_{j=1}^{n} i_{j}}$ over the feasible choices of $i_{j}$ such that $\max _{1 \leq j \leq n}\left\{i_{j}\right\} \leq n+k$. Then clearly $\eta_{n, 0} \leq \eta_{n, 1} \leq \cdots$ and $\eta_{n, k} \rightarrow \eta_{n}$ as $k \rightarrow \infty$. We claim that for every $n, k$

$$
\eta_{n, k}=\beta^{\frac{n(n+1)}{2}} \prod_{i=1}^{k} \frac{1-\beta^{n+i}}{1-\beta^{i}} .
$$

Then the limiting expression for $\eta_{n}$ follows immediately by taking $k \rightarrow \infty$.

The proof of (5) is by induction. It is trivial to validate that $\eta_{n, 0}=\beta^{\frac{n(n+1)}{2}}, n \geq 1$, and $\eta_{1, k}=\sum_{i=1}^{k+1} \beta^{i}, k \geq 0$, conform to the form of $\eta_{n, k}$ in (5). Next, we assume that (5) holds for $\eta_{i, j}$ for all $i, j$ such that either $i \leq n, j<k$ or $i<n, j \leq k$ and show that the statement is true for $\eta_{n, k}$. The quantity $\eta_{n, k}$ satisfies the following equality

$$
\eta_{n, k}=\eta_{n, k-1}+\beta^{n+k} \eta_{n-1, k}
$$

the first term corresponds to the case when $\max _{1 \leq j \leq n}\left\{i_{j}\right\} \leq n+k-1$, while the second one to the case when the last ( $n$ th) particle is in the slot $n+k$, i.e., $\max _{1 \leq j \leq n}\left\{i_{j}\right\}=n+k$. 
From (6) and the inductive assumption one derives

$$
\begin{aligned}
\eta_{n, k} & =\beta^{\frac{n(n+1)}{2}} \prod_{i=1}^{k-1} \frac{1-\beta^{n+i}}{1-\beta^{i}}+\beta^{\frac{(n-1) n}{2}+n+k} \prod_{i=1}^{k} \frac{1-\beta^{n-1+i}}{1-\beta^{i}} \\
& =\left(1+\beta^{k} \frac{1-\beta^{n}}{1-\beta^{k}}\right) \beta^{\frac{n(n+1)}{2}} \prod_{i=1}^{k-1} \frac{1-\beta^{n+i}}{1-\beta^{i}} \\
& =\beta^{\frac{n(n+1)}{2}} \prod_{i=1}^{k} \frac{1-\beta^{n+i}}{1-\beta^{i}}
\end{aligned}
$$

implying (15).

Next, we use (4) to examine the stationary deviation $\kappa_{n}$ of the last particle from its minimal position, i.e., $\kappa_{n}:=Z_{n}-n \geq 0$. Expressions (4), (15) and Lemma 2 yield

$$
\begin{aligned}
\mathbb{P}\left[\kappa_{n}=i\right] & =\frac{\eta_{n-1, i} \beta^{n+i}}{\eta_{n}} \\
& =\beta^{i}\left(1-\beta^{n}\right) \prod_{j=1}^{n-1}\left(1-\beta^{i+j}\right)<\beta^{i},
\end{aligned}
$$

and, thus,

$$
\mathbb{P}\left[\kappa_{n} \geq i\right]<(1-\beta)^{-1} \beta^{i} .
$$

Interestingly, this implies that there exists a limiting behavior for the case when the number of particles $n$ grows to infinity. While we do not use this fact for proving our main result it seems to be of independent interest. Indeed, as $n \rightarrow \infty$, the random variable $\kappa_{n}$ converges in distribution to a random variable $\kappa$, whose distribution is given as

$$
\mathbb{P}[\kappa=i]=\beta^{i} \prod_{j=1}^{\infty}\left(1-\beta^{i+j}\right) .
$$

It is interesting to observe that for $\beta<1 / 2$, or equivalently $2 q<p$, the most probable value of $\kappa$ is zero. From the preceding we conclude that $\kappa$ is asymptotically geometric with parameter $\beta$

$$
\lim _{i \rightarrow \infty} \mathbb{P}[\kappa=i] \beta^{-i}=1,
$$

and that variable $\kappa$ is stochastically monotone in parameter $\beta$.

\section{Coupling}

The following lemma relates the stationary (as $t \rightarrow \infty$ ) properties of list $L$ operating under the transposition rule and characteristics of the particle system studied in Section 3. Let $\kappa_{i}(\beta)$ explicitly denote the dependency of the random variable $\kappa_{i}$ on parameter $\beta$ (see (17)) and set $\beta:=\pi_{i+1} / \pi_{i} \leq 1$.

Proposition 1. For every $x \geq i \geq 1$

$$
\varlimsup_{t \rightarrow \infty} \mathbb{P}\left[\bigvee_{j=1}^{i} X_{j}(t)>x\right] \leq \mathbb{P}\left[\kappa_{i}(\beta)+i>x\right]
$$


Remark 1. When the support of $\pi$ is finite there exists a unique stationary solution and therefore the left side of the preceding inequality converges as $t \rightarrow \infty$.

Proof. The proof is based on a coupling argument. We start with exploiting Poisson embedding technique (see [3] for an application in the context of move-to-front rule). The requests for an item $i$, form a Poisson process of intensity $\pi_{i}$. Then, the limiting behavior (as $t \rightarrow \infty$ ) of the original discrete-time system and the system with Poisson requests is the same.

Given a Poisson process (set of arrival times) $\Lambda$ with some rate $\lambda$, let $\Lambda(p)$ denote its subset $\Lambda(p) \subseteq \Lambda$, formed by including each element of $\Lambda$ into $\Lambda(p)$ independently with probability $p$. Denote by $\Lambda_{i}$ the set of request times for item $i \in \mathbb{N}$.

Next we construct a modified list $\hat{L}$ consisting of the same items as the original one L. Parameters of the new system are denoted with the "hat" symbol. Specifically, $\hat{X}_{j}(t)$ denotes the position of element $j$ at time $t$ in the list $\hat{L}$. Each element $j \in \hat{L}$ is associated with an independent Poisson process $\hat{\Lambda}_{j}$, where

$$
\hat{\Lambda}_{j}= \begin{cases}\Lambda_{j}\left(\pi_{i} / \pi_{j}\right), & 1 \leq j \leq i, \\ \Lambda_{j} \cup \Lambda_{j}^{+}, & j>i\end{cases}
$$

where $\Lambda_{j}^{+}$is an independent Poisson process with rate $\pi_{i+1}-\pi_{j}$. Note that $\hat{\Lambda}_{j}$ are defined in such a way that they are Poisson with rates $\pi_{i}$ for $1 \leq j \leq i$ and $\pi_{i+1}$ for $j>i$. In addition, observe that $\hat{\Lambda}_{j} \subseteq \Lambda_{j}$ for $1 \leq j \leq i$ and $\hat{\Lambda}_{j} \supseteq \Lambda_{j}$ for $j>i$. Furthermore, let function $\varphi_{j}(t)$ be defined as follows

$$
\varphi_{j}(t):= \begin{cases}1_{\left\{\exists k>i: \hat{X}_{k}(t)=\hat{X}_{j}(t)-1\right\}}, & j \leq i, \\ 1_{\left\{\exists k \leq i: \hat{X}_{k}(t)=\hat{X}_{j}(t)-1\right\}}, & j>i,\end{cases}
$$

i.e., the function $\varphi_{j}(t)$ indicates whether item $j$ is preceded by an item $k$ such that rates of $\hat{\Lambda}_{j}$ and $\hat{\Lambda}_{k}$ are not equal. The request process $\hat{\Lambda}$ to list $\hat{L}$ is a superposition of Poisson processes $\hat{\Lambda}_{j}$

$$
\hat{\Lambda}(t)=\bigcup_{j: \varphi_{j}(t)=1} \hat{\Lambda}_{j}(t)
$$

Item $j$ is requested from $\hat{L}$ at time $T$ if $T \in \hat{\Lambda}_{j} \cap \hat{\Lambda}$. In other words, items $j \leq i$ are requested according to $\hat{\Lambda}_{j}$ only if they are preceded by items in $\{i+1, i+2, \ldots\}$, while the requests for items $j>i$ are placed according to $\hat{\Lambda}_{j}$ only if element $j$ is preceded by an item in $\{1, \ldots, i\}$. Note that the set of Poisson processes include in $\hat{\Lambda}$ changes with the evolution of list $\hat{L}$. In addition, the number of elements in the union in (9) is always finite and bounded from above by $2 i$.

The modified list $\hat{L}$ operates under the same transposition rule as $L$ with one modification. Namely, after the transposition rule rearranges items in either of the lists, a reordering operator $\mathcal{R}_{i}$ is applied to $\hat{L}$, which works as follows. The list is divided in two sublist: $\{1,2, \ldots, i\}$ and $\{i+1, i+2, \ldots\}$. The operator $\mathcal{R}_{i}$ reorders each sublist of $\hat{L}$ so that the order of elements within the sublists is the same as in the original list $L$ at every time. In addition only items belonging to the same sublist are allowed to exchange positions in the list. An example of how $\mathcal{R}_{i}$ operates is shown in Fig. 2

Next, assume that both lists are in the same permutation at time $t=0$. Then we argue that 


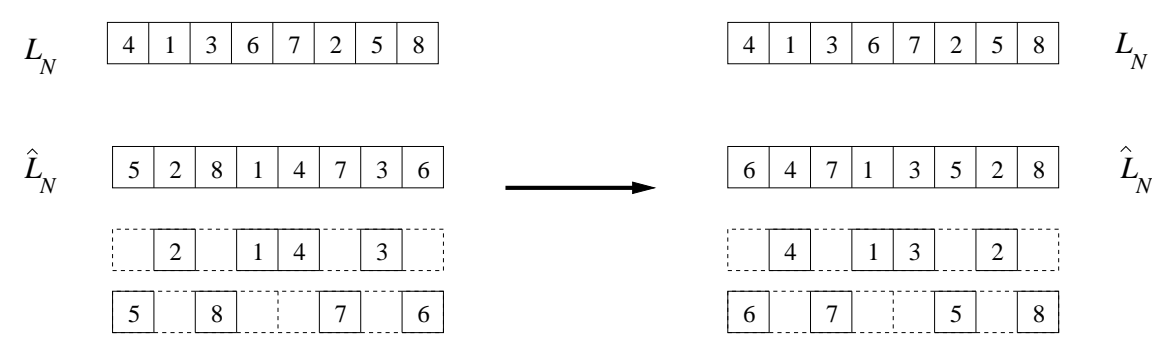

Figure 2: An example of reordering by operator $\mathcal{R}_{i}$ (in this case $i=4$ and $N=8$ ). The initial states of the lists is shown on the left. The modified list $\hat{L}_{N}$ is divided into two sublists: $\{1,2,3,4\}$ and $\{5,6,7,8\}$. Upon reordering items in each of the sublists according to $L_{N}$, the newly ordering in $\hat{L}_{N}$ is shown on the right.

Lemma 3. For every $t \in \mathbb{R}_{+}$and $1 \leq j \leq i$

$$
X_{j}(t) \leq \hat{X}_{j}(t)
$$

Proof. Either system changes only at times of requests to one of the systems; denote those times by $0<T_{1}<T_{2}<\cdots<T_{n}<\cdots$. Since there are no changes in item order between times $\left\{T_{n}\right\}_{n=1}^{\infty}$, it is sufficient to prove that (10) holds at times $\left\{T_{n}+\right\}_{n=1}^{\infty}$. To this end, assume that (10) holds for $t=T_{n-1}+$ and examine the two systems at time $t=T_{n}+$. There are three cases to examine:

(i) At time $t=T_{n}$ item $j \leq i$ is requested from $L$. By the construction of $\hat{\Lambda}$, this event implies that item $j$ is requested in the modified list $\hat{L}$ only with some probability depending on the state of $\hat{L}$.

If item $j$ is not requested from $\hat{L}$, then the set of positions occupied by items $\{1, \ldots, i\}$ in $\hat{L}$ remains the same. On the other hand, the set of positions occupied by items $\{1, \ldots, i\}$ in $L$ either does not change (when an item in $\{1, \ldots, i\}$ precedes item $j$ or $j$ is the first item in the list) or is pairwise smaller (when an item in $\{i+1, \ldots, N\}$ precedes item $j$ ). This, in conjunction with the fact that the relative order of items $1, \ldots, i$ in $L$ and $\hat{L}$ at $t=T_{n}+$ is the same (due to $\mathcal{R}_{i}$ ), implies that (10) holds for $t=T_{n}+$.

On the other hand, if item $j$ is requested in both lists, then the only case that needs to be examined in detail is the one in which $j$ is preceded by item $l \leq i$ in $L$; note that from (9) it follows that $j$ is preceded by an item $v>i$ in $\hat{L}$. Since $X_{l}\left(T_{n}-\right) \leq \hat{X}_{l}\left(T_{n}-\right)$ and the order of the items $1, \ldots, i$ is the same in both lists (due to $\mathcal{R}_{i}$ ) one has

$$
X_{j}\left(T_{n}-\right)+1 \leq \hat{X}_{j}\left(T_{n}-\right),
$$

Since the items $j$ and $l$ are transposed in $L$ but not in $\hat{L}$, the order of $j$ an $l$ is different in the two lists before $\mathcal{R}_{i}$ is applied. Thus, $\mathcal{R}_{i}$ exchanges positions of items $j$ and $l$ in $\hat{L}$. This leads to

$$
\hat{X}_{j}\left(T_{n}+\right)=\hat{X}_{l}\left(T_{n}-\right) \geq X_{l}\left(T_{n}-\right)=X_{j}\left(T_{n}+\right)
$$

and

$$
\hat{X}_{l}\left(T_{n}+\right)=\hat{X}_{j}\left(T_{n}-\right)-1 \geq X_{j}\left(T_{n}-\right)=X_{l}\left(T_{n}+\right),
$$

where the first inequality follows from the inductive assumption while the last inequality is due to (11). Thus, we conclude that (10) holds for $t=T_{n}+$. 
(ii) At time $t=T_{n}$ item $j>i$ is requested from $\hat{L}$. The argument is very similar to the one in (i). In this case $j$ is preceded by some $v \leq i$ in $\hat{L}$ (see (9)).

If item $j$ is not requested from $L$ or is preceded (in $L$ ) by an item $v>i$, then the positions occupied by items $1, \ldots, i$ in $L$ do not change. However, in $\hat{L}$ is preceded by $v \leq i$ and, thus, $v$ is moved one position back by the transposition rule.

Alternatively, if in $L$ item $j$ is requested and preceded by $l \leq i$ then either $v=l$ and $X_{l}\left(T_{n}-\right)=\hat{X}_{v}\left(T_{n}-\right)$ or $v \neq l$ and $X_{l}\left(T_{n}-\right) \leq \hat{X}_{l}\left(T_{n}-\right)+1, X_{v}\left(T_{n}-\right) \leq \hat{X}_{v}\left(T_{n}-\right)+$ 1. In either case after the items are transposed one has $X_{l}\left(T_{n}+\right) \leq \hat{X}_{l}\left(T_{n}+\right)$ and $X_{v}\left(T_{n}+\right) \leq \hat{X}_{v}\left(T_{n}+\right)$.

(iii) At time $t=T_{n}$ item $j>i$ requested from $L$ but not from $\hat{L}$. This implies that $j$ is preceded in $\hat{L}$ by an item $v>i$ (see (9)). If $j$ is preceded in $L$ by an item $l>i$ the positions occupied by items $1, \ldots, i$ do not change. Therefore, we only need to consider the case when $j$ is preceded by $l \leq i$. However, in that case we necessarily have

$$
\hat{X}_{l}\left(T_{n}-\right) \geq X_{l}\left(T_{n}-\right)+1
$$

which implies

$$
X_{l}\left(T_{n}+\right)=X_{l}\left(T_{n}-\right)+1 \leq \hat{X}_{l}\left(T_{n}-\right)=\hat{X}_{l}\left(T_{n}+\right) .
$$

The preceding (i)-(iii) establish (10).

Next, let variables $Z_{j}(t), 1 \leq j \leq i$ be defined by

$$
\left(Z_{1}(t), \ldots, Z_{i}(t)\right):=\mathcal{S}\left(\hat{X}_{1}(t), \ldots, \hat{X}_{i}(t)\right)
$$

where $\mathcal{S}$ is the sorting (in the increasing order) operator. Note that the definition of $Z_{i}(t)$ and (10) imply

$$
Z_{i}(t) \geq \bigvee_{j=1}^{i} X_{j}(t)
$$

Observe that the evolution of $\left\{Z_{j}(t)\right\}$ is probabilistically the same as in the constrained particle system described in Section 3 (recall that $Z_{j}(t)$ is the position of the $j$ th particle at time $t)$, with $p=\pi_{i} /\left(\pi_{i}+\pi_{i+1}\right), q=\pi_{i+1} /\left(\pi_{i}+\pi_{i+1}\right)$. Indeed, $Z_{j}(t)$ increases by one at Poisson rate $\pi_{i+1}$ only if $Z_{j+1}(t) \neq Z_{j}(t)+1$, while it decreases by one at rate $\pi_{i}$ only if $Z_{j-1} \neq Z_{j}(t)-1$. Next, taking maximums on both sides of (12) and then applying operator $\mathbb{P}[\cdot>x]$ leads to

$$
\mathbb{P}\left[\bigvee_{j=1}^{i} X_{j}(t)>x\right] \leq \mathbb{P}\left[Z_{i}(t)>x\right]
$$

from which one obtains

$$
\begin{aligned}
\varlimsup_{t \rightarrow \infty} \mathbb{P}\left[\bigvee_{j=1}^{i} X_{j}(t)>x\right] & \leq \mathbb{P}\left[Z_{i}>x\right] \\
& =\mathbb{P}\left[k_{i}(\beta)+i>x\right]
\end{aligned}
$$

where the last inequality follows from the definition of $\kappa_{i}(\beta)$ in Section 3 , 


\section{Proof of the main results}

Proposition 1 is the primary tool in establishing our main results. Its simple consequence is the following lemma.

Lemma 4. For any $y \geq 1$ and distribution of requests $\pi$

$$
\varlimsup_{t \rightarrow \infty} \mathbb{P}[C(t)>x] \leq \mathbb{P}\left[\kappa_{y}\left(\pi_{y+1} / \pi_{y}\right)>x-y\right]+\mathbb{P}\left[R>y, R+\kappa_{R}\left(\pi_{R+1} / \pi_{R}\right)>x\right] .
$$

Proof. Conditioning on the requested item and using the monotonicity of the max-operator result in

$$
\begin{aligned}
\varlimsup_{t \rightarrow \infty} \mathbb{P}[C(t)>x] & =\varlimsup_{t \rightarrow \infty}\left(\sum_{i \geq 1} \pi_{i} \mathbb{P}\left[X_{i}(t)>x\right]\right) \\
& \leq \sum_{i=1}^{y} \pi_{i} \varlimsup_{t \rightarrow \infty} \mathbb{P}\left[\bigvee_{j=1}^{y} X_{j}(t)>x\right]+\sum_{i \geq y+1} \pi_{i} \varlimsup_{t \rightarrow \infty} \mathbb{P}\left[\bigvee_{j=1}^{i} X_{j}(t)>x\right] \\
& \leq \mathbb{P}\left[\kappa_{y}\left(\pi_{y+1} / \pi_{y}\right)>x-y\right]+\mathbb{P}\left[R>y, R+\kappa_{R}\left(\pi_{R+1} / \pi_{R}\right)>x\right],
\end{aligned}
$$

where the last inequality follows from Proposition 1

We now complete the proofs of our main results, Theorems 1 and 2

Proof of Theorem 1 1 . The lower bound is an immediate consequence of Lemma 1 and holds for any distribution of requests $\pi$. Hence, we only consider the upper bound. We first examine the case when $\pi$ is asymptotically geometric with parameter $\nu$. Fix arbitrary small $\varepsilon>0$ such that $\nu+\varepsilon<1$. By the assumption, there exists $i_{\varepsilon}$ such that $\nu-\varepsilon<\pi_{i+1} / \pi_{i}<\nu+\varepsilon$ for all $i \geq i_{\varepsilon}$. Furthermore, setting $y=i_{\varepsilon}$ in Lemma 4 for any $s^{-1}>\nu+\varepsilon$, one obtains

$$
\begin{aligned}
\varlimsup_{t \rightarrow \infty} \mathbb{P}[C(t)>x] & \leq \mathbb{P}\left[\kappa_{i_{\varepsilon}}(\nu+\varepsilon)>x-i_{\varepsilon}\right]+\mathbb{P}\left[R+\kappa_{R}(\nu+\varepsilon)>x\right] \\
& \leq \mathbb{P}\left[s^{\kappa_{\varepsilon}(\nu+\varepsilon)}>s^{x-i_{\varepsilon}}\right]+\mathbb{P}\left[s^{R+\kappa_{R}(\nu+\varepsilon)}>s^{x}\right] \\
& \leq s^{-x} s^{i_{\varepsilon}} \mathbb{E} s^{\kappa_{i_{\varepsilon}}(\nu+\varepsilon)}+s^{-x} \mathbb{E} s^{R+\kappa_{R}(\nu+\varepsilon)},
\end{aligned}
$$

where the last step is due to Markov's inequality. From $s^{-1}>\nu+\varepsilon$ and (8) it follows $\mathbb{E} s^{R+\kappa_{R}(\nu+\varepsilon)}<\infty$. Then, (13) results in

$$
\begin{aligned}
x^{-1} \varlimsup_{t \rightarrow \infty} \log \mathbb{P}[C(t)>x] & \leq-\log s+x^{-1} \log \left(s^{i_{\varepsilon}} \mathbb{E} s^{\kappa_{i_{\varepsilon}}(\nu+\varepsilon)}+\mathbb{E} s^{R+\kappa_{R}(\nu+\varepsilon)}\right) \\
& \rightarrow-\log s,
\end{aligned}
$$

as $x \rightarrow \infty$. On the other hand, note that

$$
\begin{aligned}
\mathbb{P}[R>x] & \geq \sum_{i>x} \pi_{i_{\varepsilon}}(\nu-\varepsilon)^{i-i_{\varepsilon}} \\
& \geq \pi_{i_{\varepsilon}}(1-\nu+\varepsilon)^{-1}(\nu-\varepsilon)^{x+1-i_{\varepsilon}},
\end{aligned}
$$

implying

$$
\varliminf_{x \rightarrow \infty} x^{-1} \log \mathbb{P}[R>x] \geq \log (\nu-\varepsilon) .
$$


Combining (14) and (15), we obtain

$$
\varlimsup_{x \rightarrow \infty} \varlimsup_{t \rightarrow \infty} \frac{\log \mathbb{P}[C(t)>x]}{\log \mathbb{P}[R>x]} \leq \frac{\log s^{-1}}{\log (\nu-\varepsilon)}
$$

passing $s^{-1} \downarrow \nu+\varepsilon$ and $\varepsilon \downarrow 0$ yields the result.

Next, we consider the case when $\pi$ is power law distribution with parameter $\alpha>1$. Lemma 4 and (8) yield

$$
\begin{aligned}
\varlimsup_{t \rightarrow \infty} \mathbb{P}[C(t)>x] & \leq \mathbb{P}\left[\kappa_{y}\left(\pi_{y+1} / \pi_{y}\right)>x-y\right]+\mathbb{P}[R>y] \\
& \leq\left(1-\frac{\pi_{y+1}}{\pi_{y}}\right)^{-1}\left(\frac{\pi_{y+1}}{\pi_{y}}\right)^{x-y}+\mathbb{P}[R>y] .
\end{aligned}
$$

Letting $y=\lceil\varepsilon x / \log x\rceil$ for some $\varepsilon>0$ results in an estimate on the first term in the preceding sum, as $x \rightarrow \infty$,

$$
\left(1-\frac{\pi_{y+1}}{\pi_{y}}\right)^{-1}\left(\frac{\pi_{y+1}}{\pi_{y}}\right)^{x-y}=\alpha^{-1} e^{\alpha} y x^{-\alpha / \varepsilon}(1+o(1))
$$

while the second term can be approximated as

$$
\mathbb{P}[R>y]=\sum_{i>y} c i^{-\alpha}=O\left(y^{-\alpha+1}\right),
$$

as $x \rightarrow \infty$. Therefore, for sufficiently small constant $\varepsilon$ one has, as $x \rightarrow \infty$,

$$
\varlimsup_{t \rightarrow \infty} \mathbb{P}[C(t)>x] \leq \mathbb{P}[R>y](1+o(1)) .
$$

This completes the proof of Theorem 1

Proof of Theorem 2. The proof is very similar to the one of Theorem 1 and, thus, we omit details. Since

$$
\frac{\log \mathbb{P}\left[C_{N}>x\right]}{\log \mathbb{P}\left[R_{N}>x\right]}=\frac{\log \mathbb{P}\left[C_{N}>x\right]}{\log \mathbb{P}[R>x]} \frac{\log \mathbb{P}[R>x]}{\log \mathbb{P}\left[R_{N}>x\right]}
$$

and the upper bound on $\mathbb{P}\left[C_{N}>x\right]$ is the same as the one on $\varlimsup \mathbb{\operatorname { l i m }} \mathbb{P}[C(t)>x]$, we only need to verify that the last fraction in the preceding equality tends to one as $\{x, N\} \rightarrow \infty$. However, that easily follows from the assumptions of the theorem.

\section{Conclusions}

We have established an asymptotic tail optimality of the transposition rule in linear lists. Specifically, when the requests probability distribution is power law or geometric we showed that, when the transposition rule is used to organize the lists, the logarithm of the tail probability of the search cost is asymptotically optimal when the system has been in operation for a very large time. It would be interesting to see how quickly the limiting behavior is reached, that is how quickly the underlying Markov chain mixes. One way to approach this question would be to analyze the mixing rate of the constrained asymmetric exclusion process. 


\section{References}

[1] E. Anderson, P. Nash, and R. Weber. A counterexample to a conjecture in optimal list ordering. J. Appl. Probab., 19(9):730-732, 1982.

[2] R. Bachrach and R. El-Yaniv. Online list accessing algorithms and their applications: Recent empirical evidence. In Proc. ACM-SIAM SODA, 1997.

[3] J. Fill. An exact formula for the move-to-front rule for self-organizing lists. J. Theoret. Probab., 9(1):113-159, 1996.

[4] J. Fill. Limits and rate of convergence for the distribution of search cost under the move-to-front rule. Theoret. Comput. Sci., 164:185-206, 1996.

[5] P. Flajolet, D. Gardy, and L. Thimonier. Birthday paradox, coupon collector, caching algorithms and self-organizing search. Discrete Appl. Math., 39:207-229, 1992.

[6] J. Hester and D. Hirchberg. Self-organizing linear search. Computing Surveys, 17(3):295-311, September 1985.

[7] P. Jelenković. Asymptotic approximation of the move-to-front search cost distribution and least-recently-used caching fault probabilities. Ann. Appl. Probab., 9(2):430-464, 1999.

[8] Y. Kan and S. Ross. Optimal list order under partial memory constraints. J. Appl. Probab., 17:1004-1015, 1980.

[9] D. Knuth. The Art of Computer Programming, volume 3. Sorting and Searching. AddisonWesley, 2nd edition, 1998.

[10] K. Lam, M. Y. Leung, and M. K. Siu. Self-organizing files with dependent accesses. J. Appl. Probab., 21:343-359, 1984.

[11] R. Rivest. On self-organizing sequential search heuristics. Comm. ACM, 19(2):63-67, 1976.

[12] D. Sleator and R. Tarjan. Amortized efficiency of list update and paging rules. Comm. ACM, 28(2):202-208, 1985.

[13] A. Tenenbaum and R. Nemes. Two spectra of self-organizing sequential search algorithms. SIAM J. Comput., 11(3):557-566, 1982. 